\title{
51. Study on the Stereotactic Surgery by Localized Cooling and Freezing (Part II)
}

\author{
Yuji Miyazaki and Teruyoshi Hashiba \\ Dept. of Nurosurgery, Sapporo Medical College
}

The object of use of the localized cooling to $+3-+5^{\circ} \mathrm{C}$ is the production of temporary and reversible intracerebral lesion and this cooling reversible lesion is very valuable in stereotactic operations to find the good effective target,particularly in cases of cerebral atrophy like cerebral palsy. The area of reversible lesion made by long slender refrigeration brain probe sized in $2 \mathrm{~mm}$ in diameter by slowly is $4 \mathrm{~mm}$ in diameter.

The long slender refrigeration probe is also able to use to make a permanent lesion by freezing to below $0^{\circ} \mathrm{C}$. The fundamental studies were done on the possibility of use of the freezing method for permanent lesion.

The freezing lesion is localized, sharply circumscribed and adjacent to intact normal brain tissue in $1 \mathrm{~mm}$. from the edge of the lesion. On the other hand, the freezing lesion has great disadvantage in predictability and reproducibility. From datas of our own animal experiments in cats and dogs, the size of the freezing lesion has great deal of variation even when the freezing temperature and the duration are constant.

As Dr. Balthasar shown in 1957, some freezing lesion in brain have hemorrhage within the brain lesion itself. We have found the incidence of gross hemorrhage was greatest when the rate of freezing is rapid.

\section{Statistic Observation of the Effects of Stellectomy on Cerebral Diseases}

\author{
Hiromu Takebayashi, Ichiro Fujita, Kazuo Tsukiyama \\ and Masatsugu KISHI \\ 1st Dept. of Surgery, Wakayama Medical College
}

80 patients, aged from 6 months to 64 yrs, who had undergone stellectomy during the last 7 years (male 56, female 24) were discussed by follow up observation, and 67 of them were statistically examined as to the operative indications and effects.

The total cases were categorized in 15 epilepsies, 10 spastic hemiplegias after head injury, 12 spastic hemiplegias following cerebral vascular disturbances, 